\title{
Prevalence of Mycobacterium tuberculosis Infection in Suspected Patients in a Teaching Hospital in Northeastern Iran: A Cross-Sectional Study
}

\author{
Jamal falahi ${ }^{1,2,4}$, Kiarash Gazvini ${ }^{1}$, Marzieh Mirzaei ${ }^{3}$, Araz Majnoni ${ }^{2}$, Hadi Lotfi ${ }^{2}$ \\ $\&$ Saeid Amel Jamehdar ${ }^{1}$ \\ ${ }^{1}$ Antimicrobial Resistance Research Center, Mashhad University of Medical Sciences, Mashhad, Iran \\ ${ }^{2}$ Department of Microbiology and Virology, Faculty of Medicine, Mashhad University of Medical Sciences, \\ Mashhad, Iran \\ ${ }^{3}$ M.Sc. Student in Nursing, School of Nursing and Midwifery, Mashhad University of Medical Sciences, Mashhad, Iran \\ ${ }^{4}$ Pediatric Infections Research Center, Research Institute for Children`s Health, Shahid Beheshti University of \\ Medical Science, Tehran, Iran. \\ Correspondence: Saeid Amel Jamehdar, Antimicrobial Resistance Research Center, Mashhad University of \\ Medical Sciences, Mashhad, Iran.E-mail: ameljs@mums.ac.ir
}

Received: July 7, 2017

doi:10.5539/jmbr.v8n1p95
Accepted: July 21, 2018

Online Published: July 24, 2018

\begin{abstract}
Background: Mycobacterium tuberculosis is an infectious agent responsible for major health problems and a large number of mortalities. The prevalence of Mycobacterium tuberculosis infection varies across countries. Knowing the infection prevalence can aid in improving public health and reduce the associated costs. The aim of this study was to determine the prevalence of tuberculosis (TB) infection in suspected cases in Mashhad, Iran.
\end{abstract}

Methods: All the clinical specimens suspected of TB infection were sent to a laboratory for diagnosis during March 2017 to March 2018. The samples were analyzed microscopically using Ziehl-Neelsen staining, by polymerase chain reaction (PCR) to identify the Mycobacterium tuberculosis species using IS6110 primers, and the samples were also grown on Lowenstein-Jensen medium.

Results: Of 2,755 clinical samples analyzed, 153 (5.55\%) were identified as Mycobacterium tuberculosis-positive, of which $54.9 \%$ originated from females and $45.1 \%$ from males. The highest rate of infection was observed in spring, especially in May (15\%). Most TB cases were found in patients in VIP (43.1\%), thorax (17\%), and internal $(15 \%)$ wards. TB infection was mostly detected in bronchial tube (70\%) and sputum (23.5\%) samples. The most common positive smear was $1+(36 \%)$. Of the 153 cases, (147) $96.1 \%$ were culture -positive and 2\% were PCRnegative also $84.3 \%$ were smear - positive.

Conclusion: The highest rate of infection occurred in spring, when the number of religious tourists entering the city was at its peak. Considering the sensitive location of this city, awareness regarding TB status can lead to improved health in the community and development of basic strategies to control and eliminate the transmission of this infection from Mashhad to other areas.

Keywords: Mycobacterium tuberculosis, Prevalence, Religious city

\section{Introduction}

Tuberculosis (TB) is an airborne infectious disease caused by Mycobacterium tuberculosis. The disease is a leading cause of mortality worldwide. TB is one of the immediate priorities of the World Health Organization (WHO), especially in countries with a low socioeconomic status (de Oliveira Uehara et al., 2016; Viney et al., 2015).

The Global TB Report by the WHO in 2017 indicated that the TB epidemic remains one of the world's greatest health concerns (Dou, Chen, Kou, \& Su, 2015). Although a wide range of efforts have been made to eliminate the disease in accordance with the WHO Plan to Stop Tuberculosis 2015-1998, many countries still report high TB infection rates, particularly African and Asian countries (Organization, 2016a; Wang, Sng, \& Tay, 2004). According to the WHO, approximately 20 million people suffer from TB, with about 8 million new cases and 3 
million deaths per year. The prevalence of this disease is growing, particularly in individuals with risk factors and especially in immunocompromised patients (Asgharzadeh \& Kafil, 2007; Chaiprasert, Prammananan, Tingtoy, \& Na-Ubol, 2006; Wang et al., 2004).

The incidence of TB varies across countries, and according to reports from countries with high infection rates during 1998-2015, 22 countries account for approximately 80\% of all global infections (Organization, 2016b). Afghanistan and Uganda were removed from the list in 2017, and India and China constitute about $30 \%$ of the total (Organization, 2016b). The prevalence of TB differs not only between countries but can also differ in different provinces of a country. Diverse statistics have been reported for the incidence of TB infection in different regions of Iran, and the infection is especially prevalent in the eastern and northeastern regions, which are near endemic countries listed by the WHO (Organization, 2016a). Religious cities in Iran annually receive a large influx of visitors from neighboring countries including Afghanistan, Pakistan, Iraq, Lebanon, Syria, and Yemen. Mashhad is a major religious tourism city with the annual arrival of many tourists. The study of TB prevalence and the implementation of health surveillance play an essential role in infection control and prevention of transmission to low-incidence TB regions (Tavanaee Sani et al., 2015). We aimed to determine the prevalence of Mycobacterium tuberculosis infection in patients suspected of TB in Ghaem Teaching Hospital, Mashhad, Iran.

\section{Materials and Methods}

\subsection{Study Design}

This cross-sectional study was carried out during March 2017 to March 2018. Samples were analyzed in a comprehensive microbiology laboratory at Ghaem Teaching Hospital, in Mashhad, Khorasan Razavi province, Iran. This study was conducted because Mashhad is a religious tourism hub and millions of religious tourists visit the city annually. All the suspected cases of Mycobacterium tuberculosis infection visiting the microbiology laboratory of the hospital were enrolled, and informed consent was obtained from the patients. Broncho-alveolar, sputum, skin, and cerebrospinal fluid samples were obtained from patients in different hospital wards . Samples that were not suitable for transfer or not approved by the center expert were excluded from the study.

\subsection{Decontamination, Culture, and Staining}

All clinical specimens were decontaminated by $\mathrm{N}$-acetyl-L-cysteine- $\mathrm{NaOH}$ in a Class-2 cabin. Following decontamination, LJ medium (Biomerieuxmarcy, France) was inoculated with the samples and incubated at $37^{\circ} \mathrm{C}$ for 4-8 weeks. During this time, the Mycobacterium colonies were counted and results recorded. The decontaminated sediment from the clinical specimens was prepared on a microscopic slide using the Ziehl-Neelsen technique (Gopinath \& Singh, 2009; Li et al., 2009).

\subsection{Nucleic Acid Extraction}

DNA was extracted from clinical specimens with an RTP Mycobacteria Kit (STRATEC Molecular GmbH, BerlinBuch, Germany) according to the manufacturer's instructions. Briefly, $200 \mu \mathrm{l}$ of the clinical specimen was mixed with $200 \mu \mathrm{l}$ of NAC buffer, kept at room temperature for $20 \mathrm{~min}$, and centrifuged at $11,000 \mathrm{x}$ g. The supernatant was then mixed with $400 \mu \mathrm{l}$ of buffer $\mathrm{R}$ and transferred to an extraction tube. Other steps were performed according to the standard protocol. The extracted DNA purity and concentration were determined on a Nanodrop 1000 (Thermo Scientific). Samples were stored at $-20^{\circ} \mathrm{C}$ until further analyses.

\subsection{Polymerase Chain Reaction (PCR) Amplification}

Conventional PCR was performed using the primers listed in Table 1 on an I-cycle iQ Thermal Cycler (Bio-Rad Laboratories Inc., CA). The $20 \mu \mathrm{l}$ reactions contained $10 \mu \mathrm{l}$ of $2 \mathrm{X}$ Taq Premix including $15 \mathrm{mM} \mathrm{MgCl}_{2}, 1 \mu \mathrm{l}(0.5$ $\mathrm{pmol} / \mu \mathrm{l}$ ) of each primer, $4 \mu \mathrm{l}$ of template DNA, and $6 \mu \mathrm{l}$ of nuclease-free water (Applied Biosystems/Ambion, TX). The PCR protocol was as follows: initial denaturation at $94^{\circ} \mathrm{C}$ for $5 \mathrm{~min}$, followed by 35 cycles of denaturation at $94^{\circ} \mathrm{C}$ for $45 \mathrm{sec}$, amplification at $63^{\circ} \mathrm{C}$ for $30 \mathrm{sec}$, and extension at $72^{\circ} \mathrm{C}$ for $45 \mathrm{sec}$, followed by a final extension at $72^{\circ} \mathrm{C}$ for $5 \mathrm{~min}$. The PCR products were electrophoresed on $1.5 \%$ agarose gels.

Table 1. IS6110 primer for the detection of Mycobacterium tuberculosis

\section{Results}

\begin{tabular}{cccccc}
\hline IS6110 & Sequences & Length & TM & GC\% & Product \\
\hline Forward primer & CGTGAGGGCATCGAGGTGGC & 20 & 65.61 & 70.00 & \multirow{2}{*}{$245 \mathrm{bp}$} \\
Reverse primer & GCGTAGGCGTCGGTGACAAA & 20 & 63.35 & 60.00 & \\
\hline
\end{tabular}

Of the 153 positive cases, $69(45.1 \%)$ were male and $84(54.9 \%)$ were female. The prevalence of TB infection from the 2,755 patients was $5.55 \%$. The highest infection rate was in spring (10.5\%) and in the month of May $(15 \%)$ and the lowest rates were observed in summer and autumn. Of the hospital wards, the highest infection rates 
occurred in patients in the VIP, thorax, and internal wards with $43.1,17$, and $15 \%$, respectively, while the lowest rates occurred in the ear, nose, and throat, operating room, intensive care unit, and gynecology wards. Of the 153 positive samples, $69.9 \%$ were bronchial, $23.5 \%$ were sputum, and $0.7 \%$ were isolated from urine or abscess samples. $15.7 \%$ of the positive PCR samples were negative by microscopy, which could be due to the low sensitivity of the staining method. Of the 153 positive samples, 55 were $1+(35.9 \%)$ by microscopy, 37 were $3+$ (24.2\%), and 7(4.6\%) were $4+$. Of the 153 positive samples, 147 (\%96.1) were culture-positive and $6(3.9 \%)$ were culture-negative. The PCR results were $98 \%$ positive and $2 \%$ negative (Figs. 1-5).

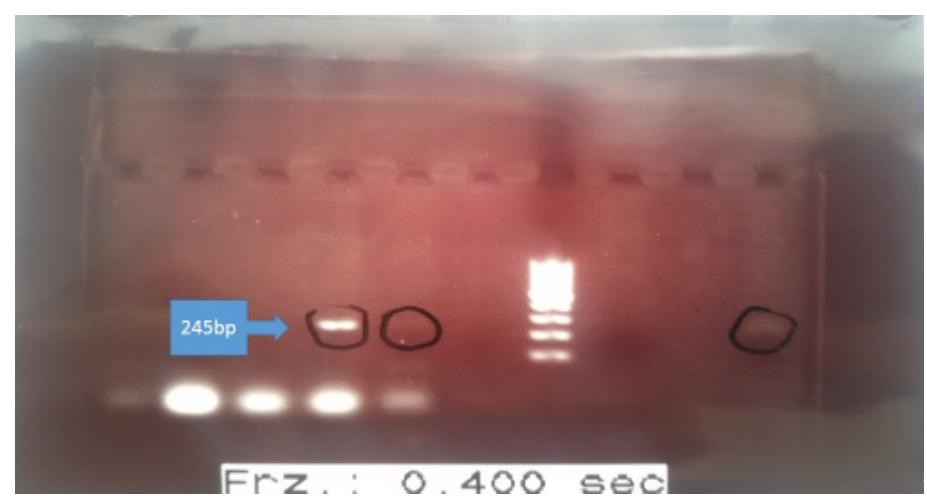

Figure 1. The 245-bp amplified product obtained from M. tuberculosis

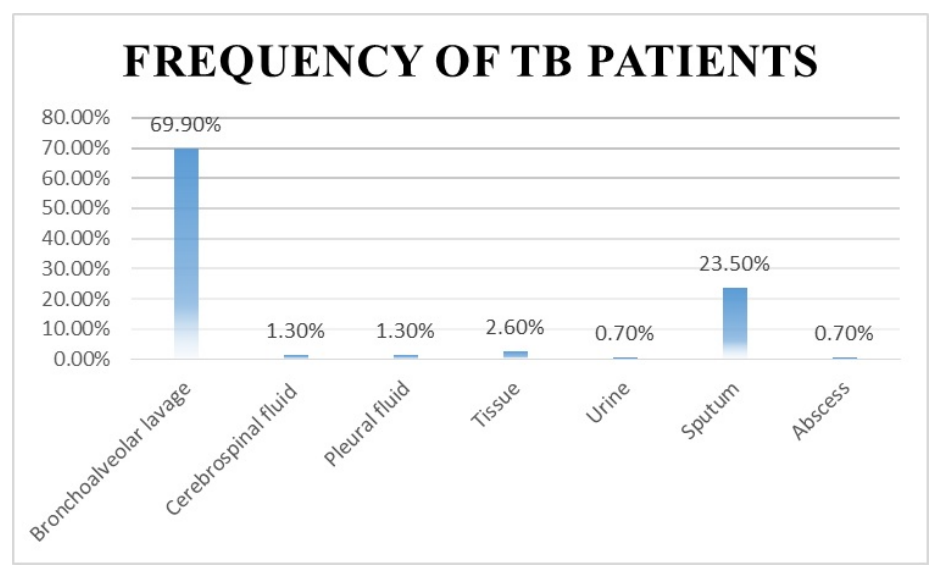

Figure 2. Tuberculosis prevalence by specimen

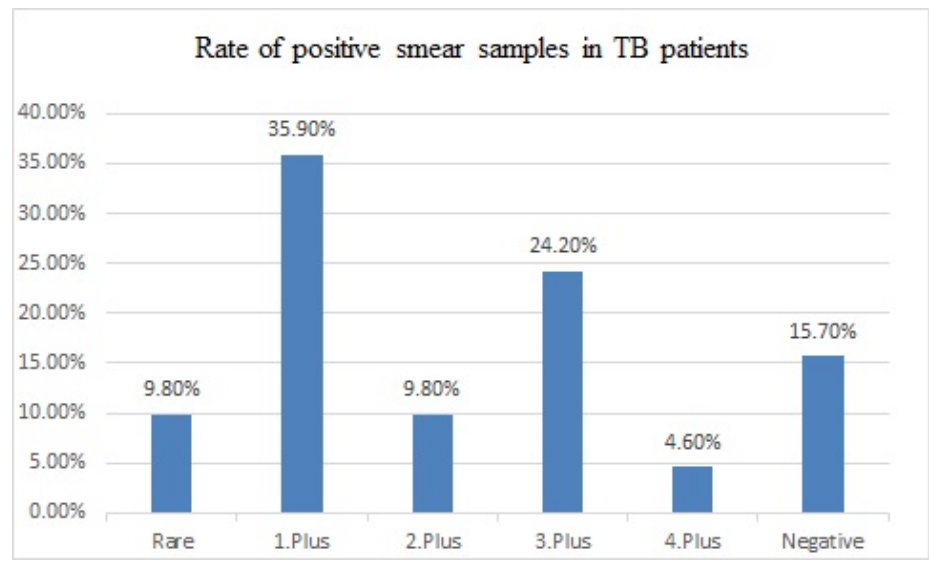

Figure 3. Rates of positive smear samples according to the American Thoracic Association 


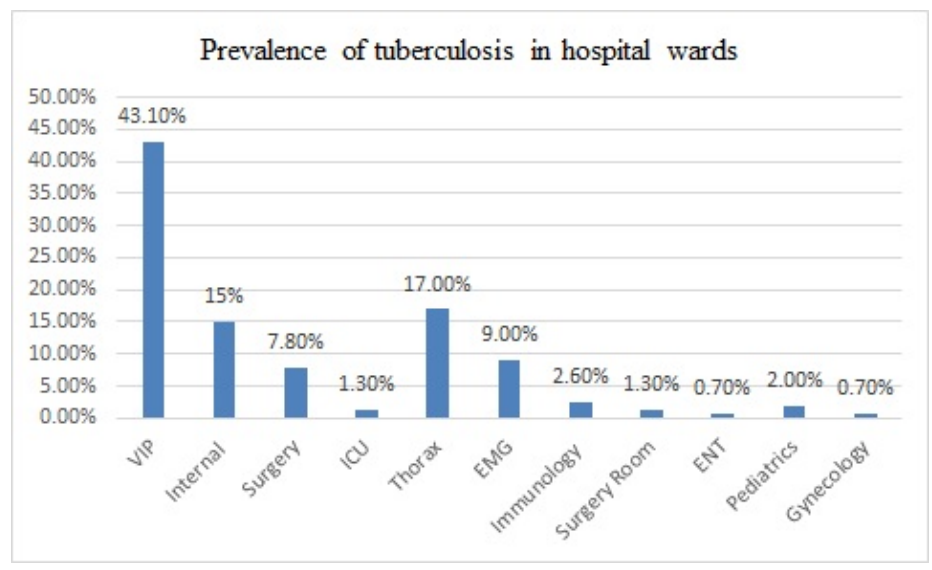

Figure 4. Tuberculosis prevalence by ward

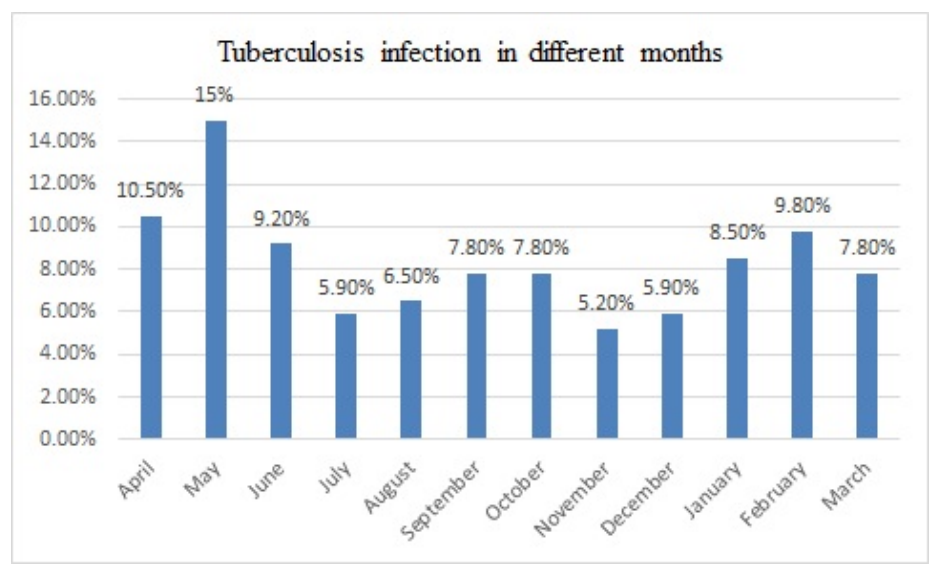

Figure 5. Tuberculosis prevalence by month

\section{Discussion}

The current study is one of the few large scale investigations, which were carried out on the prevalence determination of TB infection in Mashhad, Iran. We have demonstrated that the prevalence of TB infection was $5.55 \%(153 / 2,755)$. Also the highest prevalence of TB infection was observed in spring and summer, especially in May, which agrees with the results of other studies conducted in the north of Iran and migrant population entering Iran (Leung et al., 2005; Moosazadeh, Khanjani, \& Bahrampour, 2013; Moosazadeh, Khanjani, Bahrampour, \& Nasehi, 2014). In previous studies, the infection incidence varied in different months (Douglas, Strachan, \& Maxwell, 1996; Leung et al., 2005; Rios, Garcia, Sanchez, \& Perez, 2000). Considering that Mashhad is a religious city, the arrival of many religious tourists seems to account for the high prevalence of TB in spring and summer. The arrival of pilgrims at these times, especially from Afghanistan, Pakistan, Iraq, and Lebanon could account for the increased infection rates(RAFIEI, Besharat, JABARI, Golalipour, \& NASER, 2009).

Most of the positive samples in this study were obtained from the VIP, thoracic, and internal wards. In addition, $15 \%$ of the positive specimens were negative for Mycobacterium tuberculosis, due to Lowe sensitivity ranging from 40 to $80 \%$. Specialy lower sensitivity is encountered in pediatric TB, extrapulmonary TB and in HIVinfected TB patients, which indicates that TB infection control is essential in countries with high AIDS rates With negative sputum smears (Babady \& Wengenack, 2012; Campos, Rocha, Willers, \& Silva, 2016; Palomino, 2005).

The remarkable thing is that the TB infection rate was higher in females $(54.9 \%)$ than in males, which agrees with the findings of other studies (Alavi, Khoshkho, Salmanzadeh, \& Eghtesad, 2014; Babamahmoodi et al., 2015; Bialvaei, Asgharzadeh, Aghazadeh, Nourazarian, \& Kafil, 2017; Dhanaraj et al., 2015; Ghaffari-Fam et al., 2015; Mao et al., 2014). It seems that the greater prevalence of women in family especially crowded families with low socioeconomic status, society and other factors may contribute to this high rate, although in some studies, men 
had higher infection rates than women (Babamahmoodi et al., 2015; Bialvaei et al., 2017; Zadeh et al., 2013). Despite the relative ease of access to health care and effective drugs, TB remains a health problem in Iran, especially in the border regions (Alavi et al., 2014; Feyisa et al., 2016). Also target gene in this study was is6110, that the is 6110 gene has a variable number of copies ranging from 0 to 25 , possibly due to the lack of identification of species without is6110, the result is negatively reported(Xiong, Kong, Yang, Cheng, \& Gilbert, 2006) as In our study, of the 153 positive cases, 3 cases were reported negative, although the result of their culture was positive.

Due to its proximity with countries endemic for TB, Iran acts as a bridge for spreading TB to other countries with low TB incidence. Considering that various factors such as smoking, AIDS, drug resistance, advanced age, gender, and neighboring countries led to an increase in the prevalence and spread of TB infection to Iran, a global screening program to examine the existing status and health control policies is necessary. Limitations of the present study included lack of access to Afghan and Arab refugees residing in the province and migrants who travel continuously.

\section{Conclusion}

Performing epidemiological studies in each country and region, especially in over-crowded and tourist areas, is a strategy to manage the spread of TB. In addition, screening travelers entering these areas is an effective way to control TB transmission. Further studies on the provincial migrants are recommended.

\section{Conflicts of interest}

The authors declare no conflicts of interest.

\section{Acknowledgements}

The authors wish to appreciate the cooperation of the staff of the laboratory of microbiology of Ghaem Hospital in Mashhad, Iran.

\section{References}

Alavi, S. M., Khoshkho, M. M., Salmanzadeh, S., \& Eghtesad, M. (2014). Comparison of epidemiological, clinical, laboratory and radiological features of hospitalized diabetic and non-diabetic patients with pulmonary tuberculosis at razi hospital in ahvaz. Jundishapur Journal of Microbiology, 7(9).

Asgharzadeh, M., \& Kafil, H. S. (2007). Current trends in molecular epidemiology studies of Mycobacterium tuberculosis. Biotechnology and Molecular Biology Reviews, 2(5), 108-115.

Babady, N. E., \& Wengenack, N. L. (2012). Clinical laboratory diagnostics for Mycobacterium tuberculosis Understanding Tuberculosis-Global Experiences and Innovative Approaches to the Diagnosis: InTech.

Babamahmoodi, F., Alikhani, A., Yazdani Charati, J., Ghovvati, A., Ahangarkani, F., Delavarian, L., \& Babamahmoodi, A. (2015). Clinical epidemiology and paraclinical findings in tuberculosis patients in north of Iran. BioMed research international, 2015.

Bialvaei, A. Z., Asgharzadeh, M., Aghazadeh, M., Nourazarian, M., \& Kafil, H. S. (2017). Challenges of Tuberculosis in Iran. Jundishapur Journal of Microbiology, 10(3).

Campos, L. C., Rocha, M. V. V., Willers, D. M. C., \& Silva, D. R. (2016). Characteristics of patients with smearnegative pulmonary tuberculosis (TB) in a region with high TB and HIV prevalence. PloS one, 11(1), e0147933.

Chaiprasert, A., Prammananan, T., Tingtoy, N., \& Na-Ubol, P. (2006). One-tube multiplex PCR method for rapid identification of Mycobacterium tuberculosis. Southeast Asian journal of tropical medicine and public health, 37(3), 494.

de Oliveira Uehara, S. N., Emori, C. T., Perez, R. M., Mendes-Correa, M. C. J., de Souza Paiva Ferreira, A., de Castro Amaral Feldner, A. C., . . . Ferraz, M. L. C. G. (2016). High incidence of tuberculosis in patients treated for hepatitis C chronic infection. Brazilian Journal of Infectious Diseases, 20(2), 205-209.

Dhanaraj, B., Papanna, M. K., Adinarayanan, S., Vedachalam, C., Sundaram, V., Shanmugam, S., . . . Swaminathan, S. (2015). Prevalence and risk factors for adult pulmonary tuberculosis in a metropolitan city of South India. PloS one, 10(4), e0124260.

Dou, H.-Y., Chen, Y.-Y., Kou, S.-C., \& Su, I.-J. (2015). Prevalence of Mycobacterium tuberculosis strain genotypes in Taiwan reveals a close link to ethnic and population migration. Journal of the Formosan Medical Association, 114(6), 484-488. 
Douglas, A., Strachan, D., \& Maxwell, J. (1996). Seasonality of tuberculosis: the reverse of other respiratory diseases in the UK. Thorax, 51(9), 944-946.

Feyisa, S. G., Haeili, M., Zahednamazi, F., Mosavari, N., Taheri, M. M., Hamzehloo, G., . . Feizabadi, M. M. (2016). Molecular characterization of Mycobacterium tuberculosis isolates from Tehran, Iran by restriction fragment length polymorphism analysis and spoligotyping. Revista da Sociedade Brasileira de Medicina Tropical, 49(2), 204-210.

Ghaffari-Fam, S., Hosseini, S. R., Heydari, H., Vaseghi-Amiri, R., Daemi, A., \& Nikbakht, H. A. (2015). Epidemiological patterns of Tuberculosis disease in the Babol, Iran. Analyt Res Clin Med, 3(3), 146-149.

Gopinath, K., \& Singh, S. (2009). Multiplex PCR assay for simultaneous detection and differentiation of Mycobacterium tuberculosis, Mycobacterium avium complexes and other Mycobacterial species directly from clinical specimens. Journal of applied microbiology, 107(2), 425-435.

Leung, C. C., Yew, W. W., Chan, T. Y. K., Tam, C. M., Chan, C. Y., Chan, C. K., . . Law, W. S. (2005). Seasonal pattern of tuberculosis in Hong Kong. International journal of epidemiology, 34(4), 924-930.

Li, H., Turhan, V., Chokhani, L., Stratton, C. W., Dunbar, S. A., \& Tang, Y.-W. (2009). Identification and differentiation of clinically relevant mycobacterium species directly from acid-fast bacillus-positive culture broth. Journal of clinical microbiology, 47(12), 3814-3820.

Mao, T. E., Okada, K., Yamada, N., Peou, S., Ota, M., Saint, S., . . Pheng, S. H. (2014). Cross-sectional studies of tuberculosis prevalence in Cambodia between 2002 and 2011. Bulletin of the World Health Organization, 92(8), 573-581.

Moosazadeh, M., Khanjani, N., \& Bahrampour, A. (2013). Seasonality and temporal variations of tuberculosis in the north of Iran. Tanaffos, 12(4), 35.

Moosazadeh, M., Khanjani, N., Bahrampour, A., \& Nasehi, M. (2014). Does tuberculosis have a seasonal pattern among migrant population entering Iran? International journal of health policy and management, 2(4), 181.

Organization, W. H. (2016a). Global tuberculosis report 2016.

Organization, W. H. (2016b). Global Tuberculosis Report. Geneva: World Health Organization; 2014: WHO/HTM/TB/2012.6). Available: http://apps.who.int/iris/bitstream/10665/75938/1/9789241564502_eng. pdf.

Palomino, J. (2005). Nonconventional and new methods in the diagnosis of tuberculosis: feasibility and applicability in the field. European Respiratory Journal, 26(2), 339-350.

RAFIEI, S., Besharat, S., JABARI, A., Golalipour, F., \& NASER, M. A. (2009). Epidemiology of tuberculosis in northeast of Iran: a population-based study.

Rios, M., Garcia, J., Sanchez, J., \& Perez, D. (2000). A statistical analysis of the seasonality in pulmonary tuberculosis. European Journal of Epidemiology, 16(5), 483-488.

Tavanaee Sani, A., Shakiba, A., Salehi, M., Taghanaki, B., Reza, H., Ayati Fard, S. F., \& Ghazvini, K. (2015). Epidemiological Characterization of Drug Resistance among Mycobacterium tuberculosis Isolated from Patients in Northeast of Iran during 2012-2013. BioMed research international, 2015.

Viney, K., Hoy, D., Roth, A., Kelly, P., Harley, D., \& Sleigh, A. (2015). The epidemiology of tuberculosis in the Pacific, 2000 to 2013. Western Pacific surveillance and response journal: WPSAR, 6(3), 59.

Wang, S., Sng, L., \& Tay, L. (2004). Preliminary study on rapid identification of Mycobacterium tuberculosis complex isolates by the BD ProbeTec ET system. Journal of medical microbiology, 53(1), 57-59.

Xiong, L., Kong, F., Yang, Y., Cheng, J., \& Gilbert, G. L. (2006). Use of PCR and reverse line blot hybridization macroarray based on 16S-23S rRNA gene internal transcribed spacer sequences for rapid identification of 34 Mycobacterium species. Journal of clinical microbiology, 44(10), 3544-3550.

Zadeh, J. H., Nasehi, M., Rezaianzadeh, A., Tabatabaee, H., Rajaeifard, A., \& Ghaderi, E. (2013). Pattern of reported tuberculosis cases in iran 2009-2010. Iranian journal of public health, 42(1), 72.

\section{Copyrights}

Copyright for this article is retained by the author(s), with first publication rights granted to the journal.

This is an open-access article distributed under the terms and conditions of the Creative Commons Attribution license (http://creativecommons.org/licenses/by/4.0/). 